\title{
A CONCEPTUAL MATURITY MODEL FOR SUSTAINABLE CONSTRUCTION
}

\author{
Goh Cheng Siew ${ }^{1}$, Steve Rowlinson ${ }^{2}$
}

\begin{abstract}
The construction industry has contributed substantially to not only the growth of the economy but also the development of the environment and society. In the past decades, an increasing public awareness on the environmental and social growth has promoted the application of sustainable development in construction. Triple bottom line - economy, society and environment has been widely recognized as significant dimension for measuring the performance of sustainability. Although a number of performance indicator systems are available in the current market, few of them have considered soft systems - culture and human aspects, in measuring the performance of sustainable construction. This paper therefore aims to develop a conceptual maturity model for sustainable construction to gain a deeper and richer understanding on the actual practices on sustainable construction. Five key domains are outlined in the proposed model as the metrics with the description and sub-factors of each metric. Apart from contributing to increasing competitive advantage, the proposed model can steer the construction community to improve performance in attaining the goals of sustainable construction. Nonetheless, this conceptual maturity model is still at an early development stage and it is subject to more empirical testing and research for its practicability and further refinement.
\end{abstract}

\subsection{Introduction}

The construction industry always plays a significant role in pushing the growth of a nation, especially in its contribution to economic growth. A positive correlation is found between gross domestic product per capita and various measures of construction output (Yiu et al. 2004). The gross value of construction output often contributes approximately $4-12 \%$ of gross domestic product (GDP) to many national economies (Spence and Mulligan 1995; Presley and Meade 2010).

Notwithstanding the contribution to economic growth, the construction industry has also created substantial negative impacts on the growth of society and environment with its extensive exploitation of natural resources and the production of huge amount of waste (Chong et al. 2009; Ding 2008; Rohracher 2001; Shen and Tam, 2002; Son et al. 2011; Spence and Mulligan 1995; Tan et al. 2011). Over the years, the promotion of sustainable development has gained increasing attention due to growing public concern over environmental and social growth.

Since sustainable projects always demand additional technical expertise and initial financial investments as well as high stakeholders' commitments, the process of delivering sustainable construction projects could be more difficult than conventional projects. Furthermore, the situation becomes even more complicated when the pursuit of sustainability does not originate from the organizations' initiative. Presley and Meade (2010) pointed out that the application is sometimes initiated by the potential business values and financial incentives provided within sustainable project. Haapio and Viitaniemi (2008) also

\footnotetext{
${ }^{1} \mathrm{PhD}$ Candidate, Department of Real Estate and Construction, KB534, Knowles Building, The University of Hong Kong, Hong Kong. Email: goh11@hku.hk

${ }^{2}$ Professor of Real Estate and Construction, Department of Real Estate and Construction, KB508, Knowles Building, The University of Hong Kong, Hong Kong. Email: hrecsmr@hku.hk
} 
advocated the growing market demand and public policy have driven the implementation of sustainable construction.

It is also reported that sustainability implementation by construction practitioners arises mainly from binding legal requirements, as outlined in the regulations and legal policies to carry out their environmental duties. The government's pursuit of sustainable development has partly resulted in adjusting some elements of development and construction activities to be more socially and environmentally prudent (William and Lindsay 2007). Pearce et al (2010) also showed the adoption of different implementation plans of sustainability are being driven by the legal liability and regulations such as Occupational Safety and Health Acts (OSHA) and Erosion and Sediment Control (ESC). Although the introduction of mandatory regulations can increase the momentum of implementation drastically, adopting sustainable development in construction without a comprehensive understanding could mislead practitioners to address sustainability concerns inappropriately. Chong et al. (2009) found there is still a low knowledge level on sustainable construction and most respondents did not know how to initiate sustainability in their areas of works. A maturity model is therefore crucial to navigate a path to work towards sustainable construction holistically.

\subsection{Sustainable Construction}

Hill and Bowen (1997) described that sustainable construction was initially proposed to describe the responsibility and role of the construction industry in achieving sustainability, where the construction industry is deemed to include civil engineering and building construction. It is also important to avoid viewing sustainable construction as a site activity or a specific stage in the project life cycle. Apart from the comprehensive cycle of a construction project such as feasibility study, design, construction, operation, decommissioning, demolition and disposal, a broader concern should also be addressed on the process of creating human settlements, which entails planning, design, implementation and management (Du Plessis, 2007).

Presley and Meade (2010) also supported the view by referring to sustainable construction as not only the buildings and spaces but also the process and activity as well as the infrastructure elements such as waste management, transportation, and utility transmission systems. This paper therefore views sustainable construction as the application of sustainable development to the comprehensive construction cycle, from the extraction of raw material, through the planning, design and construction of buildings and infrastructure, until final deconstruction and management of the resultant waste (Tan et al. 2011).

\subsection{The Concept of Maturity}

Oxford dictionary defines maturity as the state, fact or period of being reached in the most advanced stage in a process. In the area of software development, Paulk et al. (1993) defined maturity as a potential growth in capability and it should also signify both the richness of an organization's software process and the consistency with which it is applied in projects throughout the organization. From the viewpoint of the organization, maturity is a state where an organization is in a perfect condition to pursue it objectives (Andersen and Jessen 2003). On one hand, Lockamy III and McCormack (2004) viewed process maturity as a process with a lifecycle assessed by the extent to which the process is explicitly defined, managed, measured and controlled with the growth in process capability, richness and consistency across the entire organization. From the risk management perspective, maturity is reflected as the sophistication of an 
organization's understanding of its risk portfolio and how to manage those risks as well as the internal business continuity systems for coping with and recovering from the eventuality (Zou et al. 2010).

Previous studies and works have demonstrated maturity as having a strong link to the most advanced stage, potential growth in capability, perfect condition, richness, consistency, and the sophistication portfolio. Hence this paper adopts the idea of maturity as the optimized capability and capacity of an organization or project against its pursued goals.

\subsection{The Development of a Maturity Model}

A maturity model is the development description of an entity or an anticipated, desired, and typical evolution path of the objects shaped as discrete stages (Becker and Knackstedt 2009; Klimko 2001). It is a certain result of applying life cycle approach where each entity develops over time until perfection is achieved (Klimko2001). The idea of a maturity model was first popularized by the "Capability Maturity Model" proposed by Software Engineering Institute of Carnegie-Mellon University between 1986 and 1993. In the work of Paulk et al. (1993), software process maturity is defined as the extent to which a specific process is explicitly defined, managed, measured, controlled and effective. In the Capability Maturity Model, five levels namely Initial (Level 1), Repeatable (Level 2), Defined (Level 3), Managed (Level 4) and Optimizing (Level 5), are defined to assess the capability of organizations against an agreed scale (Paulk et al. 1993).

Several attempts were made to expand the use of the maturity model to other disciplines and fields such as project management, organization, risk management, e-learning, service integration, the supply network and people capability (Andersen and Jessen 2002; Cooke-Davies and Arzymanow 2003; Dooley et al. 2001; Klimko 2001; Lockamy III and McCormack 2004; Zou et al. 2010). Klimko (2001) advocated the use of the maturity model in knowledge management on a comparison and benchmarking basis, where the description levels of each process to be achieved are clearly characterized. Zou et al. (2010) developed and validated a risk management maturity model (RM3) successfully and showed that the RM3 was user friendly, comprehensive, practical, and useful to gain a broad understanding of current risk management maturity in the industry. Dooley et al. (2001) also proved the positive correlation between high maturity level of new product development process with project success and organizational goals. They further put forth the notion of generalizing the maturity construct across both industrial and consumer sectors by indicating the direct relationship of maturity level with project performance. It is therefore rational to characterize a maturity model to measure holistically the degree of sustainable development achieved in construction.

\subsection{Maturity Models in Sustainable Construction}

It is crucial to understand the positioning of an organization towards sustainability for continuous improvement. Dooley et al. (2001) highlighted the importance of maturity (how well the system does what is does) and diffusion (how widely and how often the organization performs the best practice) to lead to a greater organizational effectiveness and more successful products. Examining the interactions of sustainable development and built environment throughout the production and delivery processes is critical to produce favorable outcomes in the development of sustainable construction. 
Although numerous sustainable assessment systems such as LEED, BREEAM, and Green Globes etc. have been developed, most of them limit their focus on the project level. Moreover, few of them have considered soft systems in measuring the performance of sustainable construction. Cooper (1999) found that BREEAM and other existing methods are largely restricted to an environmental protection and resource efficiency agenda, with limited utility for assessing socioeconomic issues. Additionally, some sustainable rating tools such as BREEAM, LEED, BEAM and BEPAC do not include financial issues in the evaluation framework, which contradicts the economic principles of sustainable development (Ding 2008). Kibert (2007) also suggested a detailed review on the existing sustainability performance assessment systems such as LEED as there is a lack of scientific framework underpinning the systems. Ding (2008) also pointed out that sustainable rating systems seem to be increasingly used as design guidelines even though they were not originally designed to serve for the purposes.

As addressed by Yao et al. (2011), the lack of an integrated approach reduces the concern for achieving balance between economic, social and environmental dimensions, and although some studies attempt to incorporate them into the practices, dynamic interactions of different factors have been neglected. Gladwin et al. (1997 cited in Cole 2005) also suggested emphasizing whole over constituent parts, relationship over specific entities, process and transformation over physical structure, quality over quantity, and inclusiveness over exclusiveness. Therefore, it is crucial to assess sustainability in construction comprehensively. Contrary to conventional green assessment systems, the proposed maturity model takes soft issues into consideration such as the culture and the management system as the metrics in examining sustainability performance.

Since sustainable construction involves a long term development that embraces the operational life cycle, the process should be emphasized rather than only the product itself. The development of a sustainable maturity model is hence significant in assessing the status of the sustainable development process and positioning the current performance level. By evaluating the maturity level on the achievement of sustainable development construction, one organization or project can hence know its own strength and weakness as well as the external opportunity and threat.

\subsection{Key Attributes of a Conceptual Maturity Model for Sustainable Construction}

Buildings, people and systems interact with one another and form a dynamic system which could be sensitive to small changes or perturbations of interacting factors (Lu et al. 2010). Lu et al. (2010) asserted that building environmental systems are complex dynamic systems which involve building and its systems; the processes which take place in planning, designing, constructing, and operating the building; the information and communication systems; and the end users. It is therefore important to have an integrated approach to examine the holism of the maturity status of sustainable construction by scrutinizing the external influences and the subtle internal fluctuations of the environmental, socioeconomic and cultural factors. Apart from technical content, the inclusion of criteria such as measurability, applicability, and communicability is acknowledged to be significant in developing a credible maturity model for sustainable construction.

Five domains are identified as key metrics in developing the maturity model for sustainable construction, i.e. performance, management capability and capacity, culture, long term framework development, and research and development, as shown in Figure 1. In each domain, sub factors are assigned as the attributes 
determining the level to be achieved in the maturity model. It is essential to define measurement indicators in each domain to allow future researchers building on this proposed maturity model for its validation and further refinement. A measurement scale of 5 points is used to assess the sub factors under each domain. An accumulated scoring basis will subsequently be used in assessing the maturity level of sustainability in construction. In the maturity model, a predefined maturity level ranging from 1 to 5 will be used to indicate the maturity index of sustainable construction applied in practice, where level 5 indicates the highest level of maturity status. The characteristics of five levels employed in the maturity model are summarized in Table 1.

\subsection{Domain 1: Performance}

The measurement of the performance is a strategic plan which focuses on the short term evaluation by examining the efficiency and effectiveness of applying sustainability in the construction process. The performances are generally evaluated against nine main principles of sustainable construction, as outlined in Table 2. Several sub factors are identified in each domain to measure and determine the sustainable competitiveness of the performance achieved.

Rather than serving as a definitive metric, the measuring approach should be applied with flexibility and adaptability. The achievement of performance should not be determined by the collection of the key performance indicators only but also the sustainable values of the projects and organizations, including life cycle operation, stakeholders' expectations and intangible social benefits to gain a holistic and actual view on sustainable development. It is important to note that the actual role of sustainability played in the construction industry should not be limited as a demonstration role for the public or the fulfillment of legal requirements only.

\subsection{Domain 2: Management capability and capacity}

The capacity and capability of an organization or project play an important role in the development of sustainable construction. The leadership style of a leader, the availability and the allocation of resources significantly impact the effectiveness and efficiency of the implementation especially in the formulation of strategies. Factors used as metrics in measuring the domain of management capability and capacity include i) specialized expertise, skill and knowledge, ii) technical tools and techniques, , iii) equipment and facilities, and iv) financial capability.

\subsection{Domain 3: Culture}

A success of sustainable development in the construction industry requires extensive support from the community, society, and people. Soft issues such as culture, attitude, communication and human interaction help to determine the development and the achievement of sustainable construction implementation. Cooke-Davies and Arzymanow (2003) cited the definitions of Denison (1990) by referring to culture as "the underlying beliefs, values and principles that serve as a foundation for an organization management system as well as the set of management practices and behaviors that both exemplify and reinforce the principles". Although culture is dynamic and changes over time, it can reflect the attitude of people and exert an influence on the decision making and practices performed by the construction players. A strong culture can also bring significant positive values to the organization and industry by attaining a shared vision and goal congruence among employees to meet organizational goals; empowering employees to be flexible; and energizing employees (Bharadwaj et al. 1993). 
There are four measuring components for sustainable culture, i.e. common language, awareness, concern, and self motivation. Urgency of taking action has to be included in the awareness for developing sustainability in construction. By cultivating a sustainable culture within the organizations and industry, people can share a common ground and language on the definition, principles and concepts of sustainable construction. As different organizations may have their own sustainable tools, protocols and standards, it is hence essential to have effective communication to bridge the gaps in applying sustainability in the construction practices.

\subsection{Domain 4: Development of organized and structured sustainable framework}

The success of sustainability cannot be gauged without a determined vision and mission as well as the tactical framework. It is vital to investigate whether sustainability features have been consistently built in or integrated into planning and daily practices. Additionally, a synergy with the core values of sustainability and the expectation of stakeholders is also needed to be attained. The importance of a well planned strategy for a short and long term cannot be overlooked since it is the main input to provide a direction for employees to focus in the present and future.

The process and strategy of a sustainable framework should embrace the following factors: i) a clear vision and the tangible mission; ii) data repository of previous projects; iii) sustainability audit and reporting, and iv) knowledge sharing platform. Tan et al. (2010) identified the importance of a clear sustainability strategy by allowing organizations to assume their commitments based on their respective backgrounds and situations. The database is important because it not only acts for self-reported metrics but also for knowledge sharing purposes. The database should be always kept in the company portfolio for ease of utilization whenever it is needed. As denoted by Chong et al. (2010), knowledge sharing among peers is critical to spread new sustainable knowledge viewing that sustainable construction concepts often need the crossover and integration of knowledge in different fields and areas

\subsection{Domain 5: Research and development ( $R \&$ D)}

Since sustainable construction highlights the long term development of economic, social and environment issues, a regular update of the latest information and technology can stimulate a continuous growth by keeping pace with the current sustainable trends. Additional insights into the exploration of the continuum of construction development could provide an advantage to outperform one undertaking from the competitors in the industry. The measurement criteria in the domain of research and development should include but not be limited to: i) innovation and ii) continuous learning.

\subsection{Conclusion}

With an increasing public awareness of the environmental and social benefits, sustainable development has gained momentum in the construction industry. Nonetheless, there is a lack of mechanisms for examining the extent of the implementation of sustainable construction holistically. The proposed conceptual maturity model can serve as valuable benchmarking tool in determining the achievement of an undertaking towards the development of sustainable construction. Through this model, a deeper and richer understanding of the actual practice of sustainable construction can also be gained by identifying the internal strength and weakness as well as the external opportunity and threat. Five main domains 
261 structured sustainable framework, and research and development are developed as the key measurement 262 metrics in the maturity model. The model offers an initial baseline to measure the evolution of sustainable 263 development maturity across the construction industry. Nonetheless, the model is subject to more 264 empirical testing and research for its practicability. Further contributions can be made with the in depth 265 validation of the model to transform it to a concrete tool for the construction industry. 


\section{References:}

Andersen, Erling S., and Svein Arne Jessen. "Project Maturity in Organisations." International Journal of Project Management 21, no. 6 (2003): 457-61.

Becker, Jörg, Ralf Knackstedt, and Jens Pöppelbuß. "Developing Maturity Models for It Management." [In English]. Business \& Information Systems Engineering 1, no. 3 (2009/06/01 2009): 213-22.

Bharadwaj, Sundar G., P. Rajan Varadarajan, and John Fahy. "Sustainable Competitive Advantage in Service Industries: A Conceptual Model and Research Propositions." Journal of Marketing 57, no. 4 (1993): 83-99.

Chong, Wai Kiong, Sanat Kumar, Carl T. Haas, Salwa M. A. Beheiry, Lonnie Coplen, and Marvin Oey. "Understanding and Interpreting Baseline Perceptions of Sustainability in Construction among Civil Engineers in the United States." Journal of Management in Engineering 25, no. 3 (2009): 143-54.

Cole, Raymond J. "Building Environmental Assessment Methods: Redefining Intentions and Roles." Building Research \& Information 33, no. 5 (2005/09/01 2005): 455-67.

Cooke-Davies, Terence J., and Andrew Arzymanow. "The Maturity of Project Management in Different Industries: An Investigation into Variations between Project Management Models." International Journal of Project Management 21, no. 6 (2003): 471-78.

Cooper, Ian. "Which Focus for Building Assessment Methods - Environmental Performance or Sustainability?". Building Research \& Information 27, no. 4-5 (1999/07/01 1999): 321-31.

Ding, Grace K.C. "Sustainable Construction - the Role of Environmental Assessment Tools." Journal of Environmental Management 86, no. 3 (2008): 451-64.

Dooley, Kevin, Anand Subra, and John Anderson. "Maturity and Its Impact on New Product Development Project Performance." [In English]. Research in Engineering Design 13, no. 1 (2001/08/01 2001): 23-29.

Du Plessis, Chrisna. "A Strategic Framework for Sustainable Construction in Developing Countries." Construction Management and Economics 25, no. 1 (2007/01/01 2007): 67-76.

Haapio, Appu, and Pertti Viitaniemi. "A Critical Review of Building Environmental Assessment Tools." Environmental Impact Assessment Review 28, no. 7 (2008): 469-82.

Hill, Richard C., and Paul A. Bowen. "Sustainable Construction: Principles and a Framework for Attainment." Construction Management and Economics 15, no. 3 (1997/05/01 1997): 223-39.

Klimko, Gabor. "Knowledge Management and Maturity Models: Building Common Understanding." In European Conference on Knowledge Management, edited by Dan Remenyi, 269-78. Slovania: Bled School of Management Bled, 2001.

Kibert, Charles J. "The next generation of sustainable construction."Building Research \& Information, 35, no. 6(2007):595-601. 
Lockamy III, A., and K. McCormack. "The Development of a Supply Chain Management Process Maturity Model Using the Concepts of Business Process Orientation." Supply Chain Management 9, no. 4 (2004): 272-78

Lu, Xiaoshu, Derek Clements-Croome, and Martti Viljanen. "Integration of Chaos Theory and Mathematical Models in Building Simulation: Part I: Literature Review." Automation in Construction 19, no. 4 (2010): 447-51.

Oxford Dictionary Online, s.v. "maturity", assessed January 18, 2013, http://oxforddictionaries.com/definition/english/maturity?q=maturity.

Paulk, Mark C; Curtis, Bill; Chrissis, Mary Beth; Weber, Charles V. "Capability Maturity Model, Version 1.1." IEEE Software 10, no. 4 (1993): 18-27.

Pearce, Annie R., Sushil Shenoy, Christine M. Fiori, and Zachary Winters. "The State of Sustainability Best Practices in Construction: A Benchmark Study." Journal of Green Building 5, no. 3 (2010/08/01 2010): 116-30.

Presley, Adrien, and Laura Meade. "Benchmarking for Sustainability: An Application to the Sustainable Construction Industry." Benchmarking 17, no. 3 (2010): 435-51.

Rohracher, Harald. "Managing the Technological Transition to Sustainable Construction of Buildings: A Socio-Technical Perspective." Technology Analysis \& Strategic Management 13, no. 1 (2001/03/01 2001): 137-50.

Shen, L. Y., and Vivian W. Y. Tam. "Implementation of Environmental Management in the Hong Kong Construction Industry." International Journal of Project Management 20, no. 7 (2002): 535-43.

Son, Hyojoo, Changwan Kim, Wai Kiong Chong, and Jui-Sheng Chou. "Implementing Sustainable Development in the Construction Industry: Constructors' Perspectives in the Us and Korea." Sustainable Development 19, no. 5 (2011): 337-47.

Spence, Robin, and Helen Mulligan. "Sustainable Development and the Construction Industry." Habitat International 19, no. 3 (1995): 279-92.

Tan, Yongtao, Liyin Shen, and Hong Yao. "Sustainable Construction Practice and Contractors' Competitiveness: A Preliminary Study." Habitat International 35, no. 2 (2011): 225-30.

Yao, Hong, Liyin Shen, Yongtao Tan, and Jianli Hao. "Simulating the Impacts of Policy Scenarios on the Sustainability Performance of Infrastructure Projects." Automation in Construction 20, no. 8 (2011): 1060-69.

Yiu, C. Y., X. H. Lu, M. Y. Leung, and W. X. Jin. "A Longitudinal Analysis on the Relationship between Construction Output and Gdp in Hong Kong." Construction Management and Economics 22, no. 4 (2004/05/01 2004): 339-45.

Williams, Katie, and Morag Lindsay. "The Extent and Nature of Sustainable Building in England: An Analysis of Progress." Planning Theory \& Practice 8, no. 1 (2007/03/01 2007): 31-49.

Zimmermann, M., H. J. Althaus, and A. Haas. "Benchmarks for Sustainable Construction: A Contribution to Develop a Standard." Energy and Buildings 37, no. 11 (2005): 1147-57. 
368 Zou, Patrick X. W., Chen Ying, and Chan Tsz-Ying. "Understanding and Improving Your Risk Management Capability: Assessment Model for Construction Organizations." Journal of Construction Engineering \& Management 136, no. 8 (2010): 854-63. 


\section{TABLE}

Table 1 The characteristics of maturity level in the conceptual sustainable development maturity model (adopted from Paulk et al. 1993)

\begin{tabular}{|l|l|}
\hline Maturity Level & Characteristics \\
\hline Level 1: Initial & $\begin{array}{l}\text { The organizations or the projects are characterized as ad hoc and occasionally } \\
\text { chaotic. The structure of organizations and projects are ill defined and individual } \\
\text { efforts are emphasized for the success. }\end{array}$ \\
\hline $\begin{array}{l}\text { Level 2: } \\
\text { Repeatable }\end{array}$ & $\begin{array}{l}\text { Certain processes are established to track and monitor the cost, time and } \\
\text { functionality. The necessary process discipline is applied for similar projects. }\end{array}$ \\
\hline Level 3: Defined & $\begin{array}{l}\text { The processes are documented, standardized and integrated into organization } \\
\text { practices. Rather than fixed, the processes can always be tailored to address } \\
\text { individual project needs. }\end{array}$ \\
\hline Level 4: Managed & $\begin{array}{l}\text { Detailed measures of process and products are clearly specified. Organizations can } \\
\text { quantitatively understand and control the process and products. }\end{array}$ \\
\hline $\begin{array}{l}\text { Level 5: } \\
\text { Optimizing }\end{array}$ & $\begin{array}{l}\text { Continuous improvement is enforced by monitoring feedback from the process. } \\
\text { Innovative ideas and technology will be developed. }\end{array}$ \\
\hline
\end{tabular}


Table 2 Nine (9) main principles of sustainable construction

\begin{tabular}{|c|c|}
\hline Principles & Sub Factors \\
\hline 1. Resources and Materials Consumption: & $\begin{array}{l}\text { a) Recycling and reuse of materials and waters } \\
\text { b) Resource usage efficiency } \\
\text { c) Land use }\end{array}$ \\
\hline 2. Environmental impact: & $\begin{array}{l}\text { a) Waste management, } \\
\text { b) Toxics elimination } \\
\text { c) Carbon emission } \\
\text { d) Ecosystem } \\
\text { e) Water efficiency }\end{array}$ \\
\hline 3. Quality of comfort: & $\begin{array}{l}\text { a) Occupational health and safety } \\
\text { b) Indoor environment quality (air, noise, lighting, } \\
\text { ventilation, temperature and humidity) } \\
\text { c) Indoor chemical and pollutant source control } \\
\text { d) Controllability of Systems (Lighting, temperature, } \\
\text { ventilation) } \\
\text { e) Occupants and owner's satisfaction }\end{array}$ \\
\hline 4. Energy efficiency & $\begin{array}{l}\text { a) Renewable energy (Biomass, wind energy, solar energy) } \\
\text { b) Optimum energy performance }\end{array}$ \\
\hline 5. Design Process & $\begin{array}{l}\text { a) Daylight } \\
\text { b) Thermal comfort } \\
\text { c) Ventilation } \\
\text { d) Spaces flexibility and adaptability } \\
\text { e) Ecological innovation }\end{array}$ \\
\hline 6. Life cycle costing & $\begin{array}{l}\text { a) Cost efficiency } \\
\text { b) Financial return } \\
\text { c) Payback period }\end{array}$ \\
\hline 7. Functional applicability & a) Market demand and supply \\
\hline 8. Life span & $\begin{array}{l}\text { a) Service life /durability of building and design } \\
\text { b) Maintenance and Refurbishment }\end{array}$ \\
\hline 9. Heritage and cultural preservation & $\begin{array}{l}\text { a) Heritage preservation } \\
\text { b) Cultural preservation }\end{array}$ \\
\hline
\end{tabular}




\section{FIGURE}

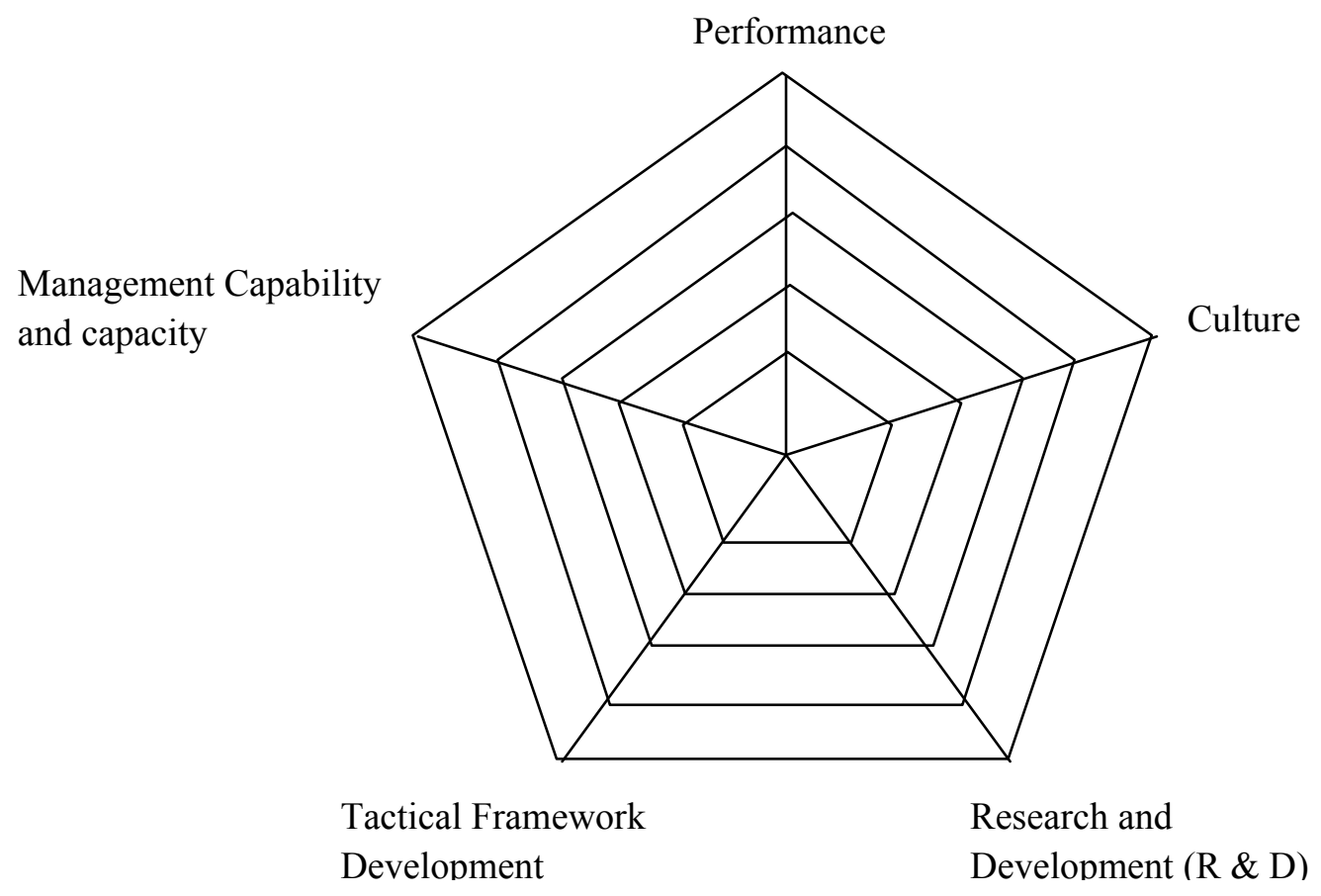

2

3

Figure 1 The Conceptual Maturity Model of Sustainable Construction 